\title{
Correction to: Effect of galcanezumab on severity and symptoms of migraine in phase 3 trials in patients with episodic or chronic migraine
}

Michael Ament ${ }^{1}$, Kathleen Day ${ }^{2 *}$, Virginia L. Stauffer ${ }^{2}$, Vladimir Skljarevski ${ }^{2}$, Mallikarjuna Rettiganti ${ }^{2}$, Eric Pearlman ${ }^{2}$ and Sheena K. Aurora ${ }^{2}$

Correction to: J Headache Pain 22, 6 (2021)

https://doi.org/10.1186/s10194-021-01215-9

Following the publication of the original article [1], the authors have notified us of a few mistakes in Table 1, marked with red below.

\section{Author details}

${ }^{1}$ Ament Headache Center, Denver, CO 80206, USA. ${ }^{2}$ Eli Lilly and Company, Indianapolis, IN 46285, USA.

Published online: 26 August 2021

\section{Reference}

1. Ament (2021) Effect of galcanezumab on severity and symptoms of

migraine in phase 3 trials in patients with episodic or chronic migraine. J

Headache Pain 63:22. https://doi.org/10.1186/s10194-021-01215-9

The original article can be found online at https://doi.org/10.1186/s10194021-01215-9.

* Correspondence: day_kathleen_ann@lilly.com

2Eli Lilly and Company, Indianapolis, IN 46285, USA

Full list of author information is available at the end of the article

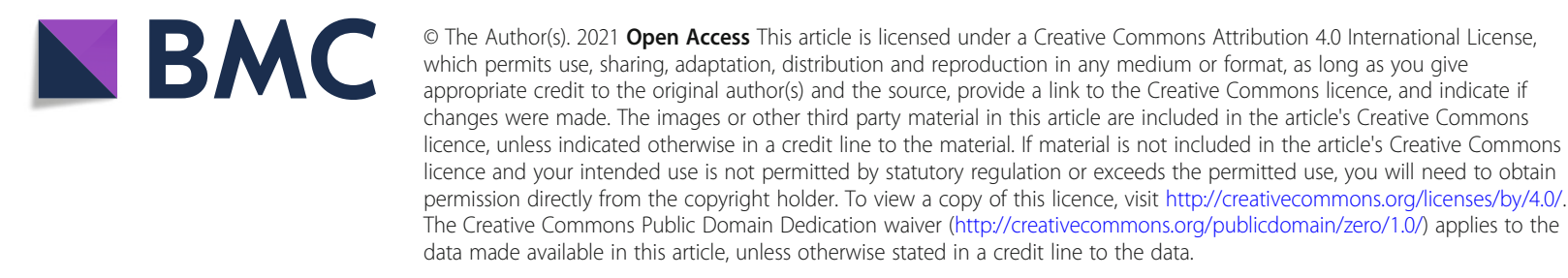


Table 1 Baseline demographics and disease characteristics

\begin{tabular}{|c|c|c|c|c|c|c|c|c|}
\hline \multirow[t]{2}{*}{ Variable } & \multicolumn{4}{|c|}{$\begin{array}{l}\text { EVOLVE-1 and EVOLVE-2 } \\
\text { (Episodic Migraine Trials) }\end{array}$} & \multicolumn{4}{|c|}{ REGAIN (Chronic Migraine Trial) } \\
\hline & $\begin{array}{l}\mathrm{PBO} \\
\mathrm{N}=894\end{array}$ & $\begin{array}{l}\text { GMB } \\
120 \\
\mathrm{mg} \\
\mathrm{N}=44 \\
4\end{array}$ & $\begin{array}{l}\text { GMB } \\
240 \\
\mathrm{mg} \\
\mathrm{N}=43 \\
5\end{array}$ & $\begin{array}{l}\text { Total } \\
\mathrm{N}=177 \\
3\end{array}$ & $\begin{array}{l}\mathrm{PBO} \\
\mathrm{N}=558\end{array}$ & $\begin{array}{l}\text { GMB } \\
120 \mathrm{mg} \\
\mathrm{N}=278\end{array}$ & $\begin{array}{l}\text { GMB } \\
240 \mathrm{mg} \\
\mathrm{N}=277\end{array}$ & $\begin{array}{l}\text { Total } \\
\mathrm{N}=1113\end{array}$ \\
\hline Age, years, mean (SD) & $\begin{array}{l}41.9 \\
(11.4) \\
\end{array}$ & $\begin{array}{l}40.9 \\
(11.5)\end{array}$ & $\begin{array}{l}40.5 \\
(11.2) \\
\end{array}$ & $\begin{array}{l}41.3 \\
(11.4) \\
\end{array}$ & $\begin{array}{l}41.6 \\
(12.1) \\
\end{array}$ & $\begin{array}{l}39.7 \\
(11.9)^{*}\end{array}$ & $\begin{array}{l}41.1 \\
(12.4)\end{array}$ & $41.0(12.1)$ \\
\hline Female, $\mathrm{n}(\%)$ & $\begin{array}{l}755 \\
(84.5)\end{array}$ & $\begin{array}{l}378 \\
(85.1)\end{array}$ & $\begin{array}{l}366 \\
(84.1)\end{array}$ & $\begin{array}{l}1499 \\
(84.6)\end{array}$ & $\begin{array}{l}483 \\
(87)\end{array}$ & $\begin{array}{l}237 \\
(85)\end{array}$ & $226(82)$ & $946(85.0)$ \\
\hline $\begin{array}{l}\text { Years since migraine } \\
\text { diagnosis, mean (SD) }\end{array}$ & $\begin{array}{l}20.5 \\
(12.5)\end{array}$ & $\begin{array}{l}20.5 \\
(12.3) \\
\end{array}$ & $\begin{array}{l}19.7 \\
(12.0) \\
\end{array}$ & $\begin{array}{l}20.3 \\
(12.4) \\
\end{array}$ & $\begin{array}{l}21.9 \\
(12.9) \\
\end{array}$ & $\begin{array}{l}20.4 \\
(12.7) \\
\end{array}$ & $\begin{array}{l}20.1 \\
(12.7) *\end{array}$ & $21.1(12.8)^{*}$ \\
\hline $\begin{array}{l}\text { Number of migraine } \\
\text { headache days/month }\end{array}$ & $\begin{array}{l}9.1 \\
(3.0) \\
\end{array}$ & $\begin{array}{l}9.1 \\
(3.0) \\
\end{array}$ & $\begin{array}{l}9.1 \\
(2.9) \\
\end{array}$ & $\begin{array}{l}9.1 \\
(3.0) \\
\end{array}$ & $19.6(4.6)$ & $\begin{array}{l}19.4 \\
(4.3) \\
\end{array}$ & $\begin{array}{l}19.2 \\
(4.6) \\
\end{array}$ & $19.4(4.5)$ \\
\hline $\begin{array}{l}\text { Number of severe } \\
\text { migraine headache } \\
\text { days/month }\end{array}$ & $\begin{array}{l}2.6 \\
(2.4)\end{array}$ & $\begin{array}{l}2.5 \\
(2.3)\end{array}$ & $\begin{array}{l}2.7 \\
(2.3)\end{array}$ & $\begin{array}{l}2.6 \\
(2.3)\end{array}$ & $6.6(5.2)$ & $6.7(4.9)$ & $6.6(4.8)$ & $6.6(5.0)$ \\
\hline $\begin{array}{l}\text { Number of moderate- } \\
\text { severe migraine } \\
\text { headache days }\end{array}$ & $\begin{array}{l}7.4 \\
(3.0)\end{array}$ & $\begin{array}{l}7.4 \\
(3.0)\end{array}$ & $\begin{array}{l}7.5 \\
(3.1)\end{array}$ & $\begin{array}{l}7.4 \\
(3.1)\end{array}$ & $16.1(5.4)$ & $\begin{array}{l}16.0 \\
(5.2)\end{array}$ & $\begin{array}{l}15.7 \\
(5.4)\end{array}$ & $16.0(5.4)$ \\
\hline $\begin{array}{l}\text { Mean severity of } \\
\text { migraine headache days }\end{array}$ & $\begin{array}{l}2.1 \\
(0.4)\end{array}$ & $\begin{array}{l}2.1 \\
(0.4)\end{array}$ & $\begin{array}{l}2.1 \\
(0.4)\end{array}$ & $\begin{array}{l}2.1 \\
(0.4)\end{array}$ & $2.2(0.4)$ & $2.2(0.4)$ & $2.2(0.4)$ & $2.2(0.4)$ \\
\hline $\begin{array}{l}\text { Number of migraine } \\
\text { headache days with aura }\end{array}$ & $\begin{array}{l}2.6(3.4 \\
) \\
\end{array}$ & $\begin{array}{l}2.3 \\
(3.1) \\
\end{array}$ & $\begin{array}{l}2.5 \\
(3.3) \\
\end{array}$ & $\begin{array}{l}2.5 \\
(3.3) \\
\end{array}$ & $5.0(6.9)$ & $5.3(7.3)$ & $4.8(7.0)$ & $5.0(7.0)$ \\
\hline $\begin{array}{l}\text { Number of migraine } \\
\text { headache days with } \\
\text { nausea and/or vomiting }\end{array}$ & $\begin{array}{l}3.8 \\
(3.2)\end{array}$ & $\begin{array}{l}3.9 \\
(3.2)\end{array}$ & $\begin{array}{l}4.0 \\
(3.2)\end{array}$ & $\begin{array}{l}3.8 \\
(3.2)\end{array}$ & $9.2(6.9)$ & $8.8(6.3)$ & $\begin{array}{l}8.1 \\
(6.5)^{*}\end{array}$ & $8.8(6.7)$ \\
\hline $\begin{array}{l}\text { Number of migraine } \\
\text { headache days with } \\
\text { photophobia and } \\
\text { phonophobia }\end{array}$ & $\begin{array}{l}6.9 \\
(3.7)\end{array}$ & $\begin{array}{l}6.8 \\
(3.8)\end{array}$ & $\begin{array}{l}6.8 \\
(3.6)\end{array}$ & $\begin{array}{l}6.9 \\
(3.7)\end{array}$ & $15.2(7.2)$ & $\begin{array}{l}14.8 \\
(7.0)\end{array}$ & $\begin{array}{l}14.0 \\
(7.6)^{*}\end{array}$ & $14.8(7.3)$ \\
\hline $\begin{array}{l}\text { Number of migraine } \\
\text { headache days with } \\
\text { prodromal symptoms }\end{array}$ & $\begin{array}{l}3.2 \\
(3.5)\end{array}$ & $\begin{array}{l}3.2 \\
(3.5)\end{array}$ & $\begin{array}{l}3.2 \\
(3.4)\end{array}$ & $\begin{array}{l}3.2 \\
(3.4)\end{array}$ & $6.8(7.3)$ & $7.3(7.5)$ & $7.1(7.3)$ & $7.0(7.4)$ \\
\hline $\begin{array}{l}\text { Proportion of migraine } \\
\text { headache days with } \\
\text { nausea and vomiting }\end{array}$ & $\begin{array}{l}40.4 \\
(30.1)\end{array}$ & $\begin{array}{l}41.8 \\
(32.0)\end{array}$ & $\begin{array}{l}42.3 \\
(30.2)\end{array}$ & $\begin{array}{l}41.2 \\
(30.6)\end{array}$ & $\begin{array}{l}46.4 \\
(31.3)\end{array}$ & $\begin{array}{l}44.7 \\
(29.3)\end{array}$ & $\begin{array}{l}41.5 \\
(30.3)\end{array}$ & $44.8(30.6)$ \\
\hline $\begin{array}{l}\text { Proportion of migraine } \\
\text { headache days with } \\
\text { photophobia and } \\
\text { phonophobia }\end{array}$ & $\begin{array}{l}74.5 \\
(30.2)\end{array}$ & $\begin{array}{l}72.8 \\
(31.8)\end{array}$ & $\begin{array}{l}73.9 \\
(30.7)\end{array}$ & $\begin{array}{l}73.9 \\
(30.7)\end{array}$ & $\begin{array}{l}76.4 \\
(29.6)\end{array}$ & $\begin{array}{l}75.0 \\
(29.7)\end{array}$ & $\begin{array}{l}71.6 \\
(32.7)\end{array}$ & $74.8(30.5)$ \\
\hline
\end{tabular}

${ }^{*} \mathrm{p} \leq 0.05$ vs $\mathrm{PBO} ; * \mathrm{*} \leq 0.01$ vs $\mathrm{PBO}$. ${ }^{\ddagger}$ Severity ratings: $1=$ mild, $2=$ moderate, $3=$ severe.

GMB, galcanezumab; PBO, placebo; SD, standard deviation.

${ }^{*} p \leq 0.05$ vs PBO; ${ }^{* *} p \leq 0.01$ vs PBO. ${ }^{\ddagger}$ Severity ratings: $1=$ mild, $2=$ moderate, $3=$ severe $G M B$ galcanezumab, $P B O$ placebo, $S D$ standard deviation 